\title{
PI Boundary Control of Linear Hyperbolic Balance Laws with Stabilization of ARZ Traffic Flow Models
}

\author{
Liguo Zhang ${ }^{\mathrm{a}, \mathrm{b}, *}$, Christophe Prieur ${ }^{\mathrm{c}}$, Junfei Qiao ${ }^{\mathrm{a}, \mathrm{b}}$ \\ ${ }^{a}$ Faculty of Information Technology, Beijing University of Technology, 100124, Beijing, China \\ ${ }^{b}$ Key Laboratory of Computational Intelligence and Intelligent Systems, 100124, Beijing, China \\ ${ }^{c}$ Univ. Grenoble Alpes, CNRS, Grenoble INP, GIPSA-lab, F-38000 Grenoble, France
}

\begin{abstract}
This paper investigates the proportional-integral (PI) boundary feedback control for the linear hyperbolic systems of balance laws which control and output measures are located at the boundaries. We address the issue of feedback stabilization by means of PI boundary controllers. By constructing a new weighted Lyapunov function, the sufficient conditions in terms of matrix inequalities are developed for the exponential stability of closed-loop systems. These results are illustrated by the linearized Aw-Rascle-Zhang (ARZ) traffic flow model. We design a PI boundary controller to stabilize the oscillations of the traffic parameters of a freeway segment and evaluate the performance with numerical simulations.
\end{abstract}

Keywords: Hyperbolic systems of balance laws, Boundary control, Lyapunov function, Exponential stability, ARZ traffic flow model.

\section{Introduction}

In this paper, we are concerned with balance laws governed by linear hyperbolic partial differential equations (PDEs). Such systems have been extensively used to model physical processes and engineering problems. The typical examples include SaintVenant equation for open channels in [1], Euler equation for gas pipes in [2], and Aw-Rascle-Zhang equation for long roads in $[3,4]$.

Boundary feedback control has been central for stabilization of the hyperbolic balance laws. Many results have established appropriate boundary conditions for linear or quasi-linear systems in $L^{2}$ or $H^{2}$ topology spaces as in [5, 6, 7, 8, 9]. Interested readers are suggested to review literature with [10].

Proportional-integral (PI) control is one of the mostly used method in engineering since its advantage is to cancel forced oscillations and attenuate load disturbances. Frequency domain is widely used to prove boundary stability of the closed-loop systems. An explicit necessary and sufficient condition is proposed in [11] for linear density-flow systems by using Laplace transformation. Similar results are developed with the operator and semi-group approach in [12]. Backstepping method is exploited in [13] to elaborate the PI-based trajectory tracking. Moreover, Lyapunov approach is considered in $[14,15,16]$ for linear, nonlinear and network hyperbolic systems of conservation laws following the idea introduced in [17]. However, only

\footnotetext{
This work is supported by the National Natural Science Foundation of China (NSFC, grant No. 61374076, No. 61533002), and the Beijing Natural Science Foundation (grant No. 1182001).

${ }^{*}$ Corresponding author

Email address: zhangliguo@bjut.edu.cn (Liguo Zhang)
}

few results appear in this direction due to the difficulties of constructing an appropriate Lyapunov candidate with PI boundary control.

Aw-Rascle-Zhang (ARZ) model, compared to scalar LWR model [18], [19], developed a velocity equation to reveal the anisotropic property of traffic dynamics. In [20], spectral analysis is applied to design boundary control for the linearized ARZ model. An integrated on-ramping metering and variable speed limit control is proposed in [21] to ARZ model by investing the boundary stabilization of positive hyperbolic systems. To deal with the model uncertainties, [22] develops the stochastic stability for Markov jumped hyperbolic systems with application to ARZ model. Recently, boundary control are developed in [23] by using the backstepping method to reduce stop-and-go oscillations for the congested traffic.

This paper constructs a new weighted Lyapunov function to prove the exponential stability of $n$-order linear hyperbolic balance laws with PI boundary control. For the best of our knowledge, existing results are focused on $2 \times 2$ systems. In [10, Section 5.4], PI boundary stabilization of linearized density-flow systems described by balance laws is provided with application to control in navigable rivers. Moreover in this later work, the source term is assumed to be stable. In our paper, we do not need this assumption on the source term. Furthermore we consider a more general linear hyperbolic system of balance laws and a practical application to freeway traffic by the linearized ARZ equation. PI boundary feedback controller is designed to stabilize the system by using integrated on-ramp metering and variable speed control. With respect to [15], we do not consider only a scalar conservation law, but rather a hyperbolic system of balance laws.

The paper is organized as follows. The statement of the PI 
boundary control for linear balance laws and the main result are given in Section 2. In Section 3 we present the proof of the main result by using the Lyapunnov techniques. Section 4 is devoted to the PI boundary stabilization of the linearized ARZ model. Some concluding remarks and possible further research lines are presented in Section 5.

Notation. $\mathbb{R}^{n}$ and $\mathbb{R}^{n \times n}$ denote the set of $n$-order vectors and $n$-order matrices. For a matrix $A, A^{\top}$ denotes the transpose, $\lambda_{\text {max }}(A)$ denotes the largest real part of eigenvalues of $A$, and for a partitioned symmetric matrix, the symbol $\star$ stands for the symmetric blocks. Given an integer $n, I_{n}$ is the identity matrix of order $n$. Given a function $g:[0, L] \rightarrow \mathbb{R}^{n}$, we define its $L^{2}$ norm as $\|g\|_{L^{2}\left((0, L) ; \mathbb{R}^{n}\right)}=\sqrt{\int_{0}^{L}|g(x)|^{2} d x}$ where $|\cdot|$ is the Euclidean norm in $\mathbb{R}^{n}$. We call $L^{2}\left((0, L) ; \mathbb{R}^{n}\right)$ the space of all measurable functions $g$ for which $\|g\|_{L^{2}\left((0, L) ; \mathbb{R}^{n}\right)}<\infty$.

\section{Preliminaries and main results}

Consider the one-dimensional $n \times n$ linear hyperbolic systems of balance laws

$$
\xi_{t}+\Lambda \xi_{x}=M \xi, \quad t \in[0, \infty), \quad x \in(0, L)
$$

where $\xi:(0, L) \times[0, \infty) \rightarrow \mathbb{R}^{n}, \Lambda \in \mathbb{R}^{n \times n}$ and $M \in \mathbb{R}^{n \times n}$. Without loss of generality, we may assume that $\Lambda$ is a diagonal matrix with non-zero real diagonal entries such that $\Lambda=\operatorname{diag}\left\{\Lambda^{+},-\Lambda^{-}\right\}$, in which $\Lambda^{+}=\operatorname{diag}\left\{\lambda_{1}, \ldots, \lambda_{m}\right\}, \Lambda^{-}=$ $\operatorname{diag}\left\{\lambda_{m+1}, \ldots, \lambda_{n}\right\}, 1 \leq m \leq n$, and $\lambda_{i}>0$ for all $i \in\{1, \ldots, n\}$.

For simplicity, we use the notations

$$
\xi_{\text {in }}(t) \triangleq\left[\begin{array}{c}
\xi^{+}(0, t) \\
\xi^{-}(L, t)
\end{array}\right], \quad \xi_{\text {out }}(t) \triangleq\left[\begin{array}{c}
\xi^{+}(L, t) \\
\xi^{-}(0, t)
\end{array}\right],
$$

to denote the input and the output of the system (1) on the left and the right boundaries, respectively, where $\xi^{+}, \xi^{-}$are defined as $\xi^{+}=\left[\xi_{1}, \ldots, \xi_{m}\right]^{\top} \in \mathbb{R}^{m}$ and $\xi^{-}=\left[\xi_{m+1}, \ldots, \xi_{n}\right]^{\top} \in \mathbb{R}^{n-m}$

In this paper, our concern is to analyze the exponential stability of the system (1) under boundary conditions of the PI feedback types, i.e.,

$$
\xi_{\text {in }}(t)=K_{p} \xi_{\text {out }}(t)+K_{I} \int_{0}^{t} \xi_{\text {out }}(\tau) d \tau,
$$

where $K_{p} \in \mathbb{R}^{n \times n}$ and $K_{I} \in \mathbb{R}^{n \times n}$ are matrix gains, and an initial condition of the form

$$
\xi(x, 0)=\xi^{0}(x), \quad x \in(0, L) .
$$

The well-posedness and the existence of the classical maximal solutions of $C^{0}\left([0, \infty) ; L^{2}\left((0, L) ; \mathbb{R}^{n}\right)\right)$ of the Cauchy problem (1)-(3) are easily adapted from [10, Appendix Theorem A.4]. The definition of the exponential stability for the system (1) is then given as follows.

Definition 1. The linear hyperbolic system (1)-(2) is exponentially stable if there exist $v>0$ and $C>0$ such that, for every $\xi^{0} \in L^{2}\left((0, L) ; \mathbb{R}^{n}\right)$, the solution to the Cauchy problem (1)-(3) satisfies

$$
\|\xi(\cdot, t)\|_{L^{2}\left((0, L) ; \mathbb{R}^{n}\right)} \leq C e^{-v t}\left\|\xi^{0}\right\|_{L^{2}\left((0, L) ; \mathbb{R}^{n}\right)},
$$

for all $t \in[0, \infty)$.
We then have the following stability theorem.

Theorem 1. The linear hyperbolic system (1)-(2) is exponentially stable if there exist a diagonal matrix $P_{1} \in \mathbb{R}^{n \times n}$, a symmetric matrix $P_{2} \in \mathbb{R}^{n \times n}$, and a matrix $P_{3} \in \mathbb{R}^{n \times n}$, a real constant $\mu$, such that the following matrix inequalities hold, for all $x \in[0, L]$

$$
\begin{aligned}
& \text { (i) } P=\left[\begin{array}{cc}
P_{1} & P_{3} \\
\star & P_{2}
\end{array}\right]>0, \\
& \text { (ii) } \Omega^{e}(x)=\left[\begin{array}{ccc}
\Omega_{11}^{e}(x) & \Omega_{12}^{e}(x) & P_{3}(x) \\
\star & \Omega_{22}^{e} & \Omega_{23}^{e} \\
\star & \star & \Omega_{33}^{e}
\end{array}\right]<0,
\end{aligned}
$$

where

$$
\begin{aligned}
& \Omega_{11}^{e}(x)=M^{\top} P_{1}(x)+P_{1}(x) M-2 \mu|\Lambda| P_{1}(x), \\
& \Omega_{12}^{e}(x)=M^{\top} P_{3}(x)-\mu|\Lambda| P_{3}(x), \\
& \Omega_{22}^{e}=\frac{1}{L}\left(K_{I}^{\top}|\Lambda| P_{1} E_{1} K_{I}+K_{I}^{\top}|\Lambda| P_{3} E_{3}+E_{3} P_{3}^{\top}|\Lambda| K_{I}\right), \\
& \Omega_{23}^{e}=\frac{1}{L}\left(K_{I}^{\top}|\Lambda| P_{1} E_{1} K_{p}-|\Lambda| P_{3} E_{4}+E_{3} P_{3}^{\top}|\Lambda| K_{p}\right)+P_{2}, \\
& \Omega_{33}^{e}=\frac{1}{L}\left(K_{p}^{\top}|\Lambda| P_{1} E_{1} K_{p}-|\Lambda| P_{1} E_{2}\right),
\end{aligned}
$$

with $P_{1}(x)=P_{1} \operatorname{diag}\left\{e^{-2 \mu x} I_{m}, e^{2 \mu x} I_{n-m}\right\}, \quad P_{3}(x)=$ $P_{3} \operatorname{diag}\left\{e^{-\mu x} I_{m}, e^{\mu x} I_{n-m}\right\}, \quad|\Lambda|=\operatorname{diag}\left\{\Lambda^{+}, \Lambda^{-}\right\}, \quad$ and $E_{1}=\operatorname{diag}\left\{I_{m}, e^{2 \mu L} I_{n-m}\right\}, E_{2}=\operatorname{diag}\left\{e^{-2 \mu L} I_{m}, I_{n-m}\right\}, E_{3}=\sqrt{E_{1}}$, $E_{4}=\sqrt{E_{2}}$.

Remark 1. It can be noticed that Theorem 1 provides a numerical tractable method to determine the gains $K_{p}$ and $K_{I}$ of the PI controller by solving the set of matrix inequalities (5), (6). This result is also consistent with the classical dissipative boundary condition for the linear hyperbolic systems of balance laws in [24], in which only the proportional feedback control is considered, as setting $K_{I}=0$.

Note that the deadbeat control obtained by letting $K_{P}=K_{I}=$ 0 stabilizes the linear hyperbolic system (1)-(2) in finite time but it is not robust with respect to small errors, in particular when the matrix $M$ is not stable. To be more specific, any small error, when numerically computing the controller, or when implementing it, destabilizes the system. In contrast, our approach is based on a Lyapunov function which guarantees a robustness and a performance of the closed-loop system.

Exploiting the linearity we can differentiate (1) with respect to $x$ and the boundary condition (2) with respect to $t$ to study the stability of the linear hyperbolic system (1)-(2) in $H^{1}$ norm. By doing so we would get similar conditions for the stability analysis in $H^{1}$ Sobolev space.

Inspired from [17], we construct a new weighted Lyapunov function to prove the exponential stabilization of the closedloop system (1)-(2) with the PI controller.

The Lyapunov function candidate $V: L^{2}\left((0, L) ; \mathbb{R}^{n}\right) \times \mathbb{R}^{n} \rightarrow$ 
$\mathbb{R}$ has the following form

$$
\begin{aligned}
V(\xi, \zeta)= & \int_{0}^{L}\left[\xi^{\top} P_{1}(x) \xi+\xi^{\top} P_{3}(x) \zeta+\zeta^{\top} P_{3}^{\top}(x) \xi\right] d x \\
& +L \zeta^{\top} P_{2} \zeta \\
= & \int_{0}^{L}\left[\begin{array}{c}
\xi \\
\zeta
\end{array}\right]^{\top}\left[\begin{array}{cc}
P_{1}(x) & P_{3}(x) \\
\star & P_{2}
\end{array}\right]\left[\begin{array}{c}
\xi \\
\zeta
\end{array}\right] d x,
\end{aligned}
$$

where $\zeta:[0, \infty) \rightarrow \mathbb{R}$ is the integral of the output of the system (1), i.e.,

$$
\zeta(t)=\int_{0}^{t} \xi_{\text {out }}(\tau) d \tau .
$$

Remark 2. The weighted Lyapunov function $V(\xi, \zeta)$ is an extension of the one $V(\xi)$ in [17], in which only the proportional feedback control is considered. The functions of the added two terms in $V(\xi, \zeta)$, which involve the integral variable $\zeta$, are to measure the energy change introduced by the integral controller.

The proof of Theorem 1 is provided in the next section.

\section{Proof of the main result}

\subsection{Proof in the case where $m=n$}

To clarify the demonstration, we first prove Theorem 1 in the special case where $m=n$, which means that the characteristic matrix $\Lambda=\Lambda^{+}$with $\lambda_{i}>0$, for all $i=1, \ldots, n$. In this case, the PI boundary controller (2) is simply rewritten as

$$
\xi(0, t)=K_{p} \xi(L, t)+K_{I} \int_{0}^{t} \xi(L, \tau) d \tau .
$$

Moreover, condition (ii) of Theorem 1 is turning into the single matrix inequality as

$$
\text { (ii') } \Omega=\left[\begin{array}{ccc}
\Omega_{11} & \Omega_{12} & P_{3} \\
\star & \Omega_{22} & \Omega_{23} \\
\star & \star & \Omega_{33}
\end{array}\right]<0,
$$

where

$$
\begin{aligned}
& \Omega_{11}=M^{\top} P_{1}+P_{1} M-2 \mu \Lambda P_{1}, \\
& \Omega_{12}=M^{\top} P_{3}-\mu \Lambda P_{3}, \\
& \Omega_{22}=\frac{1}{L}\left(K_{I}^{\top} \Lambda P_{1} K_{I}+K_{I}^{\top} \Lambda P_{3}+P_{3}^{\top} \Lambda K_{I}\right), \\
& \Omega_{23}=\frac{1}{L}\left(K_{I}^{\top} \Lambda P_{1} K_{p}-e^{-\mu L} \Lambda P_{3}+P_{3}^{\top} \Lambda K_{p}\right)+P_{2}, \\
& \Omega_{33}=\frac{1}{L}\left(K_{p}^{\top} \Lambda P_{1} K_{p}-e^{-2 \mu L} \Lambda P_{1}\right) .
\end{aligned}
$$

We assume for the time-being that the solution $\xi$ to (1) with the boundary condition (9) and the initial condition (3) is of class $C^{1}$ on $[0, L] \times[0, \infty)$. Under this assumption (that will be relaxed later on) the function $V$ defined in (7) is of class $C^{1}$, and the first step of the proof is to compute the estimation of the time-derivative $\dot{V}$ along the solution $(\xi, \zeta)$. It yields the following:

$$
\begin{aligned}
\dot{V}= & \int_{0}^{L}\left[2 \xi_{t}^{\top} P_{1} \xi e^{-2 \mu x}+\xi_{t}^{\top} P_{3} \zeta e^{-\mu x}+\zeta^{\top} P_{3}^{\top} \xi_{t} e^{-\mu x}\right. \\
& \left.+\xi^{\top} P_{3} \xi(L, t) e^{-\mu x}+\xi^{\top}(L, t) P_{3}^{\top} \xi e^{-\mu x}\right] d x \\
& +2 L \xi^{\top}(L, t) P_{2} \zeta \\
= & \dot{V}_{1}+\dot{V}_{2}+\dot{V}_{3}
\end{aligned}
$$

with

$$
\begin{aligned}
\dot{V}_{1} \triangleq 2 \int_{0}^{L} \xi_{t}^{\top} P_{1} \xi e^{-2 \mu x} d x \\
\dot{V}_{2} \triangleq \int_{0}^{L}\left[\xi_{t}^{\top} P_{3} \zeta+\zeta^{\top} P_{3}^{\top} \xi_{t}+\xi^{\top} P_{3} \xi(L, t)\right. \\
\left.\quad+\xi^{\top}(L, t) P_{3}^{\top} \xi\right] e^{-\mu x} d x \\
\dot{V}_{3} \triangleq 2 L \xi^{\top}(L, t) P_{2} \zeta .
\end{aligned}
$$

Using the PDE (1) and an integration by parts for $\dot{V}_{1}$ and $\dot{V}_{2}$, we obtain

$$
\begin{aligned}
\dot{V}_{1}= & \left.2 \int_{0}^{L}\left[-\Lambda \xi_{x}+M \xi\right)\right]^{\top} P_{1} \xi e^{-2 \mu x} d x \\
= & {\left[-\xi^{\top} \Lambda P_{1} \xi e^{-2 \mu x}\right]_{0}^{L}-2 \mu \int_{0}^{L} \xi^{\top} \Lambda P_{1} \xi e^{-2 \mu x} d x } \\
& +\int_{0}^{L} \xi^{\top}\left[M^{\top} P_{1}+P_{1} M\right] \xi e^{-2 \mu x} d x
\end{aligned}
$$

and

$$
\begin{aligned}
\dot{V}_{2}= & \int_{0}^{L}\left[-\Lambda \xi_{x}+M \xi\right]^{\top} P_{3} \zeta e^{-\mu x} d x \\
& +\int_{0}^{L} \zeta^{\top} P_{3}^{\top}\left[-\Lambda \xi_{x}+M \xi\right] e^{-\mu x} d x \\
& +\int_{0}^{L}\left[\xi^{\top} P_{3} \xi(L, t)+\xi^{\top}(L, t) P_{3}^{\top} \xi\right] e^{-\mu x} d x, \\
= & {\left[-\xi^{\top} \Lambda P_{3} \zeta e^{-\mu x}-\zeta^{\top} P_{3}^{\top} \Lambda \xi e^{-\mu x}\right]_{0}^{L} } \\
& -\mu \int_{0}^{L}\left[\xi^{\top} \Lambda P_{3} \zeta+\zeta^{\top} P_{3}^{\top} \Lambda \xi\right] e^{-\mu x} d x \\
& +\int_{0}^{L}\left[\xi^{\top} M^{\top} P_{3} \zeta+\zeta^{\top} P_{3}^{\top} M \xi\right] e^{-\mu x} d x \\
& +\int_{0}^{L}\left[\xi^{\top} P_{3} \xi(L, t)+\xi^{\top}(L, t) P_{3}^{\top} \xi\right] e^{-\mu x} d x .
\end{aligned}
$$

Then, substituting the PI boundary condition (9) into (11) and (12), we have the time-derivatives

$$
\begin{aligned}
\dot{V}_{1}= & -\xi^{\top}(L, t) \Lambda P_{1} \xi(L, t) e^{-2 \mu L} \\
& +\left[K_{p} \xi(L, t)+K_{I} \zeta\right]^{\top} \Lambda P_{1}\left[K_{p} \xi(L, t)+K_{I} \zeta\right] \\
& +\int_{0}^{L} \xi^{\top}\left[M^{\top} P_{1}+P_{1} M-2 \mu \Lambda P_{1}\right] \xi e^{-2 \mu x} d x
\end{aligned}
$$




$$
\begin{aligned}
& =\left[\begin{array}{c}
\zeta \\
\xi(L, t)
\end{array}\right]^{\top}\left[\begin{array}{c}
K_{I}^{\top} \Lambda P_{1} K_{I} \\
\star
\end{array}\right. \\
& \left.\begin{array}{c}
K_{I}^{\top} \Lambda P_{1} K_{p} \\
K_{p}^{\top} \Lambda P_{1} K_{p}-e^{-2 \mu L} \Lambda P_{1}
\end{array}\right]\left[\begin{array}{c}
\zeta \\
\xi(L, t)
\end{array}\right] \\
& +\int_{0}^{L} \xi^{\top}\left[M^{\top} P_{1}+P_{1} M-2 \mu \Lambda P_{1}\right] \xi e^{-2 \mu x} d x \\
& =\int_{0}^{L}\left[\begin{array}{c}
\xi e^{-\mu x} \\
\zeta \\
\xi(L, t)
\end{array}\right]^{\top}\left[\begin{array}{c}
M^{\top} P_{1}+P_{1} M-2 \mu \Lambda P_{1} \\
\star \\
\star
\end{array}\right. \\
& \left.\begin{array}{cc}
0 & 0 \\
\frac{K_{I}^{\top} \Lambda P_{1} K_{I}}{L} & \frac{K_{I}^{\top} \Lambda P_{1} K_{p}}{L} \\
\star & \frac{K_{p}^{\top} \Lambda P_{1} K_{p}}{L}-\frac{e^{-2 \mu L} \Lambda P_{1}}{L}
\end{array}\right]\left[\begin{array}{c}
\xi e^{-\mu x} \\
\zeta \\
\xi(L, t)
\end{array}\right] d x,
\end{aligned}
$$

and

$$
\begin{aligned}
& \dot{V}_{2}=-\xi^{\top}(L, t) \Lambda P_{3} \zeta e^{-\mu L}-\zeta^{\top} P_{3}^{\top} \Lambda \xi(L, t) e^{-\mu L} \\
& +\left[K_{p} \xi(L, t)+K_{I} \zeta\right]^{\top} \Lambda P_{3} \zeta+\zeta^{\top} P_{3}^{\top} \Lambda\left[K_{p} \xi(L, t)+K_{I} \zeta\right] \\
& -\mu \int_{0}^{L}\left[\xi^{\top} \Lambda P_{3} \zeta+\zeta^{\top} P_{3}^{\top} \Lambda \xi\right] e^{-\mu x} d x \\
& +\int_{0}^{L}\left[\xi^{\top} M^{\top} P_{3} \zeta+\zeta^{\top} P_{3}^{\top} M \xi\right] e^{-\mu x} d x \\
& +\int_{0}^{L}\left[\xi^{\top} P_{3} \xi(L, t)+\xi^{\top}(L, t) P_{3}^{\top} \xi\right] e^{-\mu x} d x \\
& =\left[\begin{array}{c}
\zeta \\
\xi(L, t)
\end{array}\right]^{\top}\left[\begin{array}{c}
K_{I}^{\top} \Lambda P_{3}+P_{3}^{\top} \Lambda K_{I} \\
\star
\end{array}\right.
\end{aligned}
$$

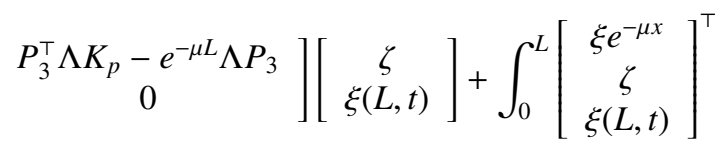

$$
\begin{aligned}
& \times\left[\begin{array}{ccc}
0 & M^{\top} P_{3}-\mu \Lambda P_{3} & P_{3} \\
\star & 0 & 0 \\
\star & \star & 0
\end{array}\right]\left[\begin{array}{c}
\xi e^{-\mu x} \\
\zeta \\
\xi(L, t)
\end{array}\right] d x \\
& =\int_{0}^{L}\left[\begin{array}{c}
\xi e^{-\mu x} \\
\zeta \\
\xi(L, t)
\end{array}\right]^{\top}\left[\begin{array}{cc}
0 & M^{\top} P_{3}-\mu \Lambda P_{3} \\
\star & \frac{K_{I}^{\top} \Lambda P_{3}}{L}+\frac{P_{3}^{\top} \Lambda K_{I}}{L} \\
\star & \star
\end{array}\right.
\end{aligned}
$$

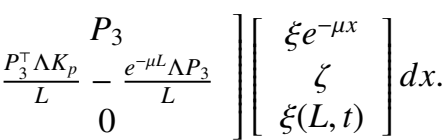

Moreover, the time-derivative $\dot{V}_{3}$ may be rewritten in the integral form as

$$
\dot{V}_{3}=\int_{0}^{L}\left[\begin{array}{c}
\xi e^{-\mu x} \\
\zeta \\
\xi(L, t)
\end{array}\right]^{\top}\left[\begin{array}{ccc}
0 & 0 & 0 \\
\star & 0 & P_{2} \\
\star & \star & 0
\end{array}\right]\left[\begin{array}{c}
\xi e^{-\mu x} \\
\zeta \\
\xi(L, t)
\end{array}\right] d x .
$$

Combining $\dot{V}_{1}$ in (13), $\dot{V}_{2}$ in (14), and $\dot{V}_{3}$ in (15) together, the time-derivative of $V(\xi, \zeta)$ satisfies

$$
\begin{aligned}
\dot{V}= & \int_{0}^{L}\left[\begin{array}{c}
\xi e^{-\mu x} \\
\zeta \\
\xi(L, t)
\end{array}\right]^{\top} \\
& \times\left[\begin{array}{ccc}
\Omega_{11} & \Omega_{12} & P_{3} \\
\star & \Omega_{22} & \Omega_{23} \\
\star & \star & \Omega_{33}
\end{array}\right]\left[\begin{array}{c}
\xi e^{-\mu x} \\
\zeta \\
\xi(L, t)
\end{array}\right] d x .
\end{aligned}
$$

It follows from the condition ( $\left.i i^{\prime}\right)$ that $\Omega<0$, then there exists a constant $v=-\frac{\lambda_{\max }(\Omega)}{\lambda_{\max }(P)}>0$ such that

$$
\begin{aligned}
\dot{V} & \leq \lambda_{\max }(\Omega) \int_{0}^{L}\left[\begin{array}{c}
\xi e^{-\mu x} \\
\zeta \\
\xi(L, t)
\end{array}\right]^{\top}\left[\begin{array}{c}
\xi e^{-\mu x} \\
\zeta \\
\xi(L, t)
\end{array}\right] d x \\
& \leq-\nu V(\xi, \zeta) .
\end{aligned}
$$

By remarking the definition of $V(\xi, \zeta)$, and coming from the condition (i) that $P>0$, we have $V(\xi, \zeta)>0$ for any classical solutions of (1)-(2). On the other hand, there exist $\beta>0$ (depending on $\left.P_{i}, i=1,2,3\right)$ such that the following inequalities hold

$$
\begin{aligned}
\frac{1}{\beta}\left(\int_{0}^{L}|\xi|^{2} d x+|\zeta|^{2}\right) & \leq V(\xi, \zeta) \\
& \leq \beta\left(\int_{0}^{L}|\xi|^{2} d x+|\zeta|^{2}\right) .
\end{aligned}
$$

Estimates (17) and (18) are obtained under the assumption that $\xi$ is of class $C^{1}$ on $[0, L] \times[0, \infty)$. But the selection of $\beta$ and $v$ does not depend on the $C^{1}$-norm of $\xi$ : they depend only on the $C^{0}\left([0, \infty) ; L^{2}\left((0, L) ; \mathbb{R}^{n}\right)\right)$-norm of $\xi$. Hence, using a classical density argument (see e.g., [10, Section 2.1.3]), the estimates (17) and (18) remain valid in the distribution sense if $\xi$ is only of class $C^{0}$.

Let $\xi \in C^{0}\left([0, \infty) ; L^{2}\left((0, L) ; \mathbb{R}^{n}\right)\right)$ be the maximal solution of the Cauchy problem (1)-(2). Using estimates (17) and (18) for all $t \in[0, \infty)$, we get that

$$
\begin{aligned}
\|\xi(\cdot, t)\|_{L^{2}\left((0, L) ; \mathbb{R}^{n}\right)} & \leq \int_{0}^{L}|\xi|^{2} d x+|\zeta|^{2} \\
& \leq \beta V(\xi, \zeta) \\
& \leq \beta^{2} e^{-v t}\left(\int_{0}^{L}\left|\xi^{0}\right|^{2} d x+|\zeta(0)|^{2}\right) \\
& =\beta^{2} e^{-v t}\left\|\xi^{0}\right\|_{L^{2}\left((0, L) ; \mathbb{R}^{n}\right)},
\end{aligned}
$$

Consequently, the hyperbolic system (1)-(2) is exponentially stable in $L^{2}$-norm by choosing $C=\beta^{2}$.

This concludes the proof of Theorem 1 in the case $m=n$.

Remark 3. Depending on the relaxation matrix $M$ being stable or not, the variable $\mu$ included in the matrix $\Omega$ might be positive or negative in Theorem 1. In fact, it is helpful to reduce the conservative of the design of the boundary feedback controller by sufficiently exploiting the relaxation structure of source terms as considered by [6].

\subsection{Proof in the case where $0<m<n$.}

In this section, we modify the proof of Theorem 1 in Section 3.1 to deal with the case $0<m<n$. The case $m=0$ is equivalent to the case $m=n$ by considering $\xi(L-x, t)$ instead of $\xi(x, t)$. 
The major difference lies in the time-derivatives $\dot{V}_{1}, \dot{V}_{2}$ which are now rewritten, respectively, as follows:

$$
\begin{aligned}
& \dot{V}_{1}=\int_{0}^{1}\left[\begin{array}{c}
\xi \\
\zeta \\
\xi_{\text {out }}
\end{array}\right]^{\top}\left[\begin{array}{c}
M^{\top} P_{1}(x)+P_{1}(x) M-2 \mu|\Lambda| P_{1}(x) \\
\star \\
\star
\end{array}\right. \\
& \left.\begin{array}{cc}
0 & 0 \\
\frac{K_{I}^{\top}|\Lambda| P_{1} E_{1} K_{I}}{L} & \frac{K_{I}^{\top}|\Lambda| P_{1} E_{1} K_{p}}{L} \\
\star & \frac{K_{p}^{\top}|\Lambda| P_{1} E_{1} K_{p}}{L}-\frac{|\Lambda| P_{1} E_{2}}{L}
\end{array}\right]\left[\begin{array}{c}
\xi \\
\zeta \\
\xi_{\text {out }}
\end{array}\right] d x,
\end{aligned}
$$

and

$$
\begin{array}{r}
\dot{V}_{2}=\int_{0}^{1}\left[\begin{array}{c}
\xi \\
\zeta \\
\xi_{\text {out }}
\end{array}\right]^{\top}\left[\begin{array}{cc}
0 & M^{\top} P_{3}(x)-\mu|\Lambda| P_{3}(x) \\
\star & \frac{K_{I}^{\top}|\Lambda| P_{3} E_{3}}{L}+\frac{E_{3} P_{3}^{\top}|\Lambda| K_{I}}{L} \\
\star & \star \\
P_{3}(x) & \\
& \frac{E_{3} P_{3}^{\top}|\Lambda| K_{p}}{L}-\frac{|\Lambda| P_{3} E_{4}}{L} \\
0
\end{array}\right]\left[\begin{array}{c}
\xi \\
\zeta \\
\xi_{\text {out }}
\end{array}\right] d x,
\end{array}
$$

where the matrices $E_{i}, i=1,2,3,4$, are defined in the statement of Theorem 1. Since $P>0$ and $\Omega^{e}(x)<0$, it may be checked that Theorem 1 can be established for the case $0<m<n$ in a manner completely parallel to the one that we have followed in the case $m=n$.

\section{PI boundary control for ARZ traffic flow model}

\subsection{ARZ Model and Linearization}

The traffic dynamics in a freeway segment are given by the so-called Aw-Rascle-Zhang equations, $x \in(0, L)$

$$
\left\{\begin{array}{l}
\partial_{t} \rho+\partial_{x}(v \rho)=0 \\
\partial_{t} v+\left(v-\rho p^{\prime}(\rho)\right) \partial_{x} v=\frac{V(\rho)-v}{\tau}
\end{array}\right.
$$

where $\rho(x, t)$ is the vehicle density, $v(x, t)$ is the average speed, $\tau$ is the relaxation time related to driving behavior. The variable $p(\rho)$ is the traffic pressure defined as

$$
p(\rho)=v_{f}-V(\rho)
$$

where $v_{f}$ is the free flow speed.

The speed-density fundamental diagram $V(\rho)$ is given in the form of Greenshields model in [25]

$$
V(\rho)=v_{f}\left(1-\left(\frac{\rho}{\rho_{m}}\right)^{\gamma}\right),
$$

where $\rho_{m}$ is the maximum density and constant $\gamma>0$.

Let $z=v, w=v+v_{f}\left(\frac{\rho}{\rho_{m}}\right)^{\gamma}$, then the nonlinear equation (22) may be written in the characteristic Riemann coordinates as

$$
\left\{\begin{array}{l}
\partial_{t} w+z \partial_{x} w=\frac{v_{f}-w}{\tau} \\
\partial_{t} z+[(1+\gamma) z-\gamma w] \partial_{x} z=\frac{v_{f}-w}{\tau}
\end{array}\right.
$$

where the sign of the velocity $(1+\gamma) z-\gamma w$ (positive or negative), indicates the freeway traffic lies in the free-flow or in the congested mode, as in [22]. We assume that the system (25) is strictly hyperbolic.

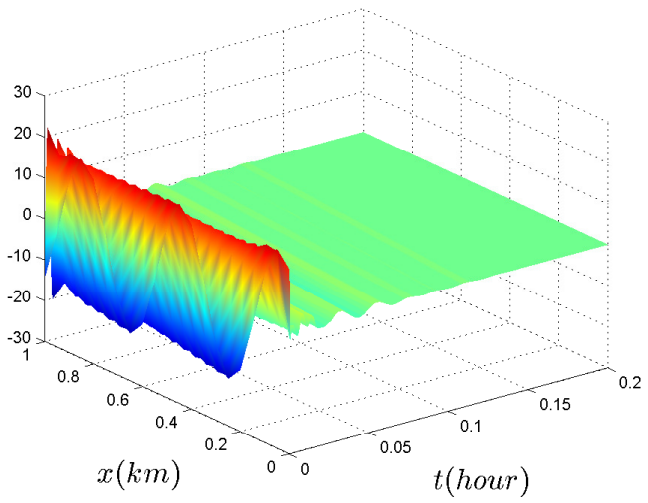

Figure 1: The evolution of the deviation $\tilde{w}$ of the linearized system (28) with PI boundary control (36).

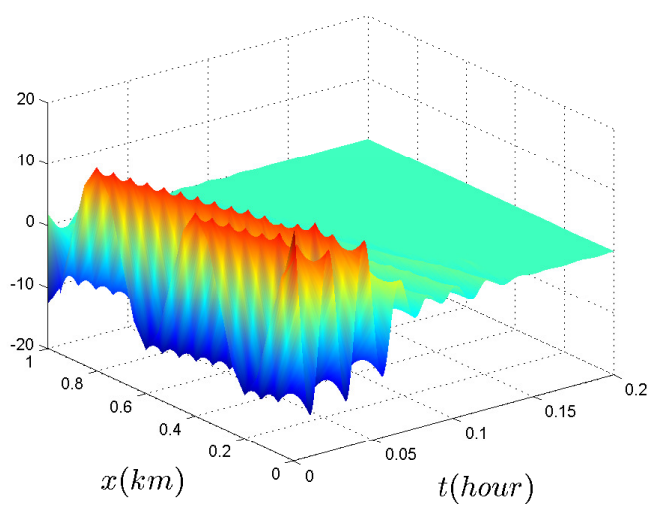

Figure 2: The evolution of the deviation $\tilde{z}$ of the linearized system (28) with PI boundary control (36).

Denote $\left(w^{*}, z^{*}\right)$ being the steady state of the system (25), the corresponding $\left(\rho^{*}, v^{*}\right)$ of the system (22), satisfying $w^{*}=v_{f}$ or function $v^{*}=V\left(\rho^{*}\right)$. The deviations from the nominal states $\left(w^{*}, z^{*}\right)$ are defined as

$$
\begin{aligned}
& \tilde{w}(x, t)=w(x, t)-w^{*}, \\
& \tilde{z}(x, t)=z(x, t)-z^{*} .
\end{aligned}
$$

We then obtain the linearized model

$$
\begin{aligned}
\partial_{t}\left[\begin{array}{c}
\tilde{w} \\
\tilde{z}
\end{array}\right] & +\left[\begin{array}{cc}
z^{*} & 0 \\
0 & (1+\gamma) z^{*}-\gamma w^{*}
\end{array}\right] \partial_{x}\left[\begin{array}{c}
\tilde{w} \\
\tilde{z}
\end{array}\right] \\
& =\left[\begin{array}{cc}
-1 / \tau & 0 \\
-1 / \tau & 0
\end{array}\right]\left[\begin{array}{c}
\tilde{w} \\
\tilde{z}
\end{array}\right] .
\end{aligned}
$$

\subsection{PI boundary feedback control}

As the freeway traffic lies in the congested regime, i.e. $(1+$ $\gamma) z^{*}-\gamma w^{*}<0$, for the boundary feedback control, the inflow speed $v(0, t)$ and outflow density $\rho(L, t)$ are measured, and the inlet on-ramp metering $r(t)$ and the outlet speed limit $v(0, t)$ are control units. 
Precisely, we introduce the PI boundary feedback law:

$$
\begin{array}{r}
r(t)=r^{*}+k_{p}^{r}\left(\rho(L, t)-\rho^{*}\right) \\
+k_{i}^{r} \int_{0}^{t}\left(\rho(L, \tau)-\rho^{*}\right) d \tau, \\
v(L, t)=v^{*}+k_{p}^{v}\left(v(0, t)-v^{*}\right) \\
+k_{i}^{v} \int_{0}^{t}\left(v(0, \tau)-v^{*}\right) d \tau,
\end{array}
$$

where $r^{*}$ is the nominal flux rate that satisfies the equilibrium relationship $p_{\text {in }}+r^{*}=\rho^{*} v^{*}$, and $k_{p}^{r}, k_{i}^{r}, k_{p}^{v}$ and $k_{i}^{v}$ are tuning gains.

Let $\tilde{\rho}=\rho-\rho^{*}, \tilde{v}=v-v^{*}$, and from the PI controller (29)-(30) directly, we have

$$
\begin{aligned}
& r(t)=r^{*}+k_{p}^{r} \tilde{\rho}(L, t)+k_{i}^{r} \int_{0}^{t} \tilde{\rho}(L, \tau) d \tau, \\
& \tilde{v}(L, t)=k_{p}^{v} \tilde{v}(0, t)+k_{i}^{v} \int_{0}^{t} \tilde{v}(0, \tau) d \tau .
\end{aligned}
$$

Using the flow-conserving condition at the inlet of the upstream boundary, i.e., $x=0$, we have

$$
p_{\text {in }}+r(t)=\rho(0, t) v(0, t) .
$$

After the linearization of the flow-conserving condition (33) with the integrating of the feedback control laws (29)-(30), we have the following boundary condition:

$$
k_{p}^{r} \tilde{\rho}(L, t)+k_{i}^{r} \int_{0}^{t} \tilde{\rho}(L, \tau) d \tau=v^{\star} \tilde{\rho}(0, t)+\rho^{\star} \tilde{v}(0, t) .
$$

As further assume $\gamma=1$, and $\alpha=\frac{v_{f}}{\rho_{m}}$, and deleting the highorder terms, we could rewrite the condition (34) in the Riemann coordinates as

$$
\begin{aligned}
\tilde{w}(0, t)= & \tilde{v}(0, t)+\alpha \tilde{\rho}(0, t) \\
= & \left(1-\frac{\alpha \rho^{\star}}{v^{\star}}\right) \tilde{v}(0, t)+\frac{\alpha k_{p}^{r}}{v^{\star}} \tilde{\rho}(L, t)+\frac{\alpha k_{i}^{r}}{v^{\star}} \int_{0}^{t} \tilde{\rho}(L, \tau) d \tau \\
= & \frac{k_{p}^{r}}{v^{\star}} \tilde{w}(L, t)+\frac{k_{i}^{r}}{v^{\star}} \int_{0}^{t} \tilde{w}(L, \tau) d \tau \\
& +\left(1-\frac{\alpha \rho^{\star}}{v^{\star}}-\frac{k_{p}^{r} k_{p}^{v}}{v^{\star}}\right) \tilde{v}(0, t) \\
& -\left(\frac{k_{p}^{r} k_{i}^{v}}{v^{\star}}+\frac{k_{i}^{r} k_{p}^{v}}{v^{\star}}\right) \int_{0}^{t} \tilde{v}(0, \tau) d \tau .
\end{aligned}
$$

Combining (30) and (35) together for the system (28), we have the following PI boundary feedback control

$$
\left[\begin{array}{c}
\tilde{w}(0, t) \\
\tilde{z}(L, t)
\end{array}\right]=K_{p}\left[\begin{array}{c}
\tilde{w}(L, t) \\
\tilde{z}(0, t)
\end{array}\right]+K_{I} \int_{0}^{t}\left[\begin{array}{c}
\tilde{w}(L, \tau) \\
\tilde{z}(0, \tau)
\end{array}\right] d \tau,
$$

where

$$
K_{p}=\left[\begin{array}{cc}
\frac{k_{p}^{r}}{v^{\star}} & 1-\frac{\alpha \rho^{\star}}{v^{\star}}-\frac{k_{p}^{r} k_{p}^{v}}{v^{\star}} \\
0 & k_{p}^{v}
\end{array}\right]
$$

and

$$
K_{I}=\left[\begin{array}{cc}
\frac{k_{i}^{r}}{v^{\star}} & \frac{-k_{p}^{r} k_{i}^{v}-k_{i}^{r} k_{p}^{v}}{v^{\star}} \\
0 & k_{i}^{v}
\end{array}\right] .
$$

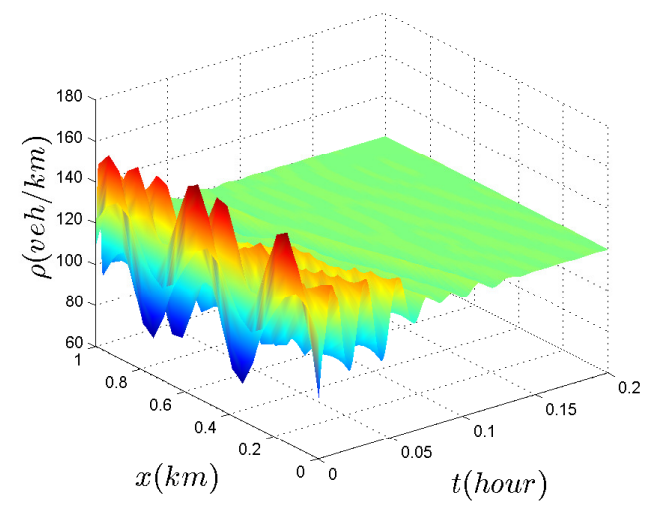

Figure 3: The evolution of $\rho$ of the nonlinear ARZ model (22) with PI boundary control (29)-(30) and steady-state $\left(\rho^{*}, v^{*}\right)=(120,70)$.

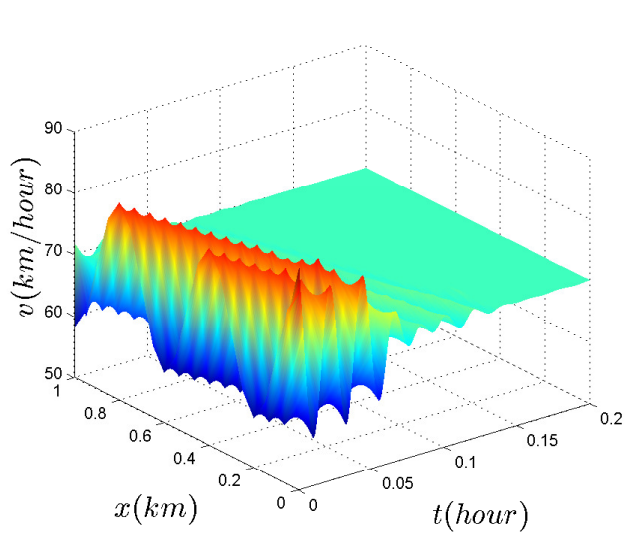

Figure 4: The evolution of $v$ of the nonlinear ARZ model (22) with PI boundary control (29)-(30) and steady-state $\left(\rho^{*}, v^{*}\right)=(120,70)$. 


\subsection{Simulations}

The developed PI boundary feedback control laws (29)-(30) for the stabilization of freeway traffic are now tested with the numerical simulation.

To this end, we consider a freeway segment whose road parameters are given, respectively, as $\rho_{m}=200 \mathrm{veh} . / \mathrm{km}, v_{f}=150$ $\mathrm{km} /$ hour, $a=0.75, p_{\text {in }}=6600$ veh./hour, $r^{*}=1800 \mathrm{veh} . /$ hour. The total road length $L=1 \mathrm{~km}$ and the relation time $\tau=60$ second.

Given the steady-state $\left(\rho^{*}, v^{*}\right)=(120,70)$, which satisfies the ARZ equations (22) with $\lambda_{1}^{*}=-20>0$. Choose the tuning parameters in (29)-(30) as $k_{i}^{v}=-0.2, k_{p}^{v}=0.1, k_{i}^{r}=-2.5$, and $k_{p}^{r}=7$. The associated boundary condition matrices $K_{p}$ and $K_{i}$ are given, respectively, as

$$
\begin{aligned}
K_{p} & =\left[\begin{array}{cc}
0.1000 & -0.0814 \\
0 & 0.1000
\end{array}\right], \\
K_{I} & =\left[\begin{array}{cc}
-0.0357 & 0.0236 \\
0 & -0.2000
\end{array}\right] .
\end{aligned}
$$

Taking $\mu=0.01$ and solving the inequality conditions (i)-(ii) of Theorem 1, we obtain the following diagonal matrix $P_{1}$, symmetric matrix $P_{2}$, and matrix $P_{3}$

$$
\begin{aligned}
P_{1} & =\left[\begin{array}{cc}
0.0610 & 0 \\
\star & 0.7755
\end{array}\right], \\
P_{2} & =\left[\begin{array}{cc}
2.1817 & 0.6366 \\
\star & 1.3823
\end{array}\right], \\
P_{3} & =\left[\begin{array}{ll}
0.0375 & 0.0094 \\
0.1604 & 0.3192
\end{array}\right] .
\end{aligned}
$$

To compute the numerical solutions of the system (28), we discretize them using the two-step variant of Lax-Wendroff method in [26]. The initial deviations from the steady-state $\left(\rho^{*}, v^{*}\right)$ are given as

$$
\left\{\begin{array}{l}
\rho(x, 0)=\rho^{*}+8 \sin (5 \pi x) \\
v(x, 0)=v^{*}+12 \cos (5 \pi x)
\end{array}\right.
$$

Figs. 1 and 2 show the time evolutions of the derivative components $\tilde{w}, \tilde{z}$, of the linearized system (28), respectively. Figs. 3 and 4 show the evolution of the vehicle density $\rho(x, t)$ and the average speed $v(x, t)$ of the nonlinear ARZ model (22) with PI boundary feedback control (29)-(30). It is observed that both variables converge to their steady-state $\rho^{*}=120 \mathrm{veh} . / \mathrm{km}$, and $v^{*}=70 \mathrm{~km} /$ hour, and almost arrive in the finite time about 0.2 hour, as expected from Theorem 1.

\section{Conclusion}

We have addressed the issue of the PI boundary control of the linear hyperbolic balance laws. The main contribution is the Lyapunov stability analysis of the proposed closed-loop system. In Theorem 1, the sufficient conditions given as a set of matrix inequalities can be used to choice the proper tuning parameters of the boundary condition. The efficiency of the theory contribution is illustrated with the boundary stabilization of the linearized ARZ model. The future research will focus on the extension of Theorem 1 to the nonuniform case and quasilinear hyperbolic systems in the $H^{2}$ space.

\section{References}

[1] V. Dos Santos, C. Prieur, Boundary control of open channels with numerical and experimental validations, IEEE Transactions on Control Systems Technology 16 (6) (2008) 1252-1264.

[2] M. Gugat, M. Dick, G. Leugering, Gas flow in fan-shaped networks: classical solutions and feedback stabilization, SIAM Journal on Control and Optimization 49 (5) (2011) 2101-2117.

[3] A. Aw, M. Rascle, Resurrection of 'second order' models of traffic flow, SIAM Journal on Applied Mathematics 60 (3) (2000) 916-938.

[4] H. M. Zhang, A non-equilibrium traffic model devoid of gas-like behavior, Transportation Research Part B 36 (3) (2002) 275-290.

[5] M. Herty, W.-A. Yong, Feedback boundary control of linear hyperbolic systems with relaxation, Automatica 69 (2016) 12-17.

[6] L. Zhang, C. Prieur, J. Qiao, Local exponential stabilization of semi-linear hyperbolic systems by means of a boundary feedback control, IEEE Control Systems Letters 2 (1) (2018) 55-60.

[7] G. Bastin, J.-M. Coron, Exponential stability of semi-linear onedimensional balance laws, in: N. Petit (Ed.), Feedback Stabilization of Controlled Dynamical Systems: In Honor of Laurent Praly, Springer, 2017, pp. 265-278.

[8] A. Hasan, O. M. Aomo, M. Krstic, Boundary observer design for hyperbolic pde-ode cascade systems, Automatica 68 (2016) 75-86.

[9] N. Espitia, A. Girard, N. Marchand, C. Prieur, Event-based control of linear hyperbolic systems of conservation laws, Automatica 70 (2016) 275-287.

[10] G. Bastin, J.-M. Coron, Stability and Boundary Stabilization of 1-D Hyperbolic Systems, Progress in Nonlinear Differential Equations and Their Applications, Springer, 2016.

[11] G. Bastin, J.-M. Coron, S. O. Tamasoiu, Stability of linear density-flow hyperbolic systems under PI boundary control, Automatica 53 (1) (2015) $37-42$.

[12] C. Z. Xu, G. Sallet, Multivariable boundary PI control and regulation of a fluid flow system, Math. Control Relat. Fields 4 (2014) 501-520.

[13] P. O. Lamare, N. Bekiaris-Liberis, Control of $2 \times 2$ linear hyperbolic systems: Backstepping-based trajectory generation and PI-based tracking, Systems \& Control Letters 86 (2015) 24-33.

[14] V. Dos Santos, G. Bastin, J.-M. Coron, B. d'Andréa Novel, Boundary control with integral action for hyperbolic systems of conservation laws: stability and experiments, Automatica 44 (5) (2008) 1310-1318.

[15] N. T. Trinh, V. Andrieu, C. Z. Xu, Design of integral controllers for nonlinear systems governed by scalar hyperbolic partial differential equations, IEEE Transactions on Automatic Control 62 (9) (2017) 4527-4536.

[16] N. T. Trinh, V. Andrieu, C. Z. Xu, Stability and output regulation for a cascaded network of $2 \times 2$ hyperbolic systems with PI control, Automatica (2018) in press.

[17] J.-M. Coron, B. d'Andréa Novel, G. Bastin, A strict Lyapunov function for boundary control of hyperbolic systems of conservation laws, IEEE Transactions on Automatic Control 52 (1) (2007) 2-11.

[18] M. J. Lighthill, J. B. Whitham, On kinematic waves. ii. a theory of traffic flow on long crowded roads, Proc. R. Soc. A (1955) 317-345.

[19] P. I. Richards, Shock waves on the highway, Oper. Res. (1956) 42-51.

[20] F. Belletti, M. Huo, X. Litrico, A. M. Bayen, Prediction of traffic convective instability with spectral analysis of the Aw-Rascle-Zhang model, Physics Letters A 379 (38) (2015) 2319-2330.

[21] L. Zhang, C. Prieur, Necessary and sufficient conditions on the exponential stability of positive hyperbolic systems, IEEE Transactions on Automatic Control 62 (7) (2017) 3610-3617.

[22] L. Zhang, C. Prieur, Stochastic stability of Markov jump hyperbolic systems with application to traffic flow control, Automatica 86 (2017) 29-37.

[23] H. Yu, M. Krstic, Traffic congestion control on Aw-Rascle-Zhang model: Full-state feedback, in: Proceedings of the American Control Conference, Milwaukee, United States, 2018.

[24] A. Diagne, G. Bastin, J.-M. Coron, Lyapunov exponential stability of 1D linear hyperbolic systems of balance laws, Automatica 48 (1) (2012) 109-114.

[25] B. D. Greenshields, A study of traffic capacity, in: Proceedings of the Highway Research Board, 1935, pp. 448-477.

[26] L. Shampine, Solving hyperbolic PDEs in Matlab, Appl. Numer. Anal. \& Comput. Math. 2 (2005) 346-358. 